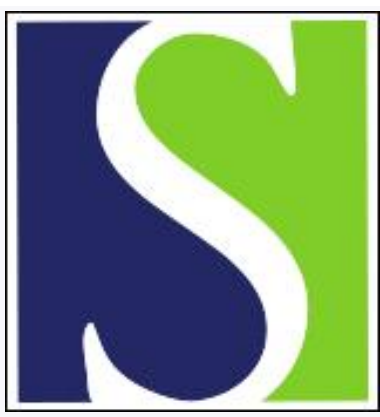

Scand J Work Environ Health 2019;45(3):318-319

https://doi.org/10.5271/sjweh.3821

Published online: 04 Apr 2019, Issue date: 01 May 2019

Response to "Development and implementation of interventions managing work-related musculoskeletal disorders: Inadequacy of prevalent research framework and future opportunities"

by van $\operatorname{der}$ Beek AJ, Coenen P

Affiliation: Department of Public and Occupational Health, Amsterdam UMC, location VUmc, van der Boechorststraat 7, 1081 BT Amsterdam, The Netherlands. a.vanderbeek@vumc.nl

Refers to the following texts of the Journal: 2019;45(3):316-317 2017;43(6):526-539 2015;41(5):491-503

Key terms: ergonomic intervention; ergonomics; evidence; intervention; musculoskeletal disorder; research framework

This article in PubMed: www.ncbi.nlm.nih.gov/pubmed/31041995 


\section{Response to "Development and implementation of interventions managing work-related musculoskeletal disorders: Inadequacy of prevalent research framework and future opportunities"}

In their recent letter to the Editor of the Scandinavian Journal of Work, Environment \& Health, Winkel \& Westgaard reflected on our earlier paper (2), where we described a research framework for the development and implementation of interventions preventing work-related musculoskeletal disorders (MSD). Winkel \& Westgaard argue that interventions described in our paper, such as manual handling advise, ergonomic workplace design, or job rotation, " ... are unlikely to have much impact" since rationalization of production systems has a larger potential in the causal pathway to health effects. Furthermore, they stress that ergonomic interventions realized as spinoff from the 'all-embracing process' of rationalization of production systems should be evaluated by using either observational or quasi-experimental research designs rather than randomized controlled trials (RCT).

We agree with Winkel \& Westgaard that effects of system changes induced by company management generally go beyond those from standalone ergonomic adjustments in the workplace and that implementation of interventions largely occurs at company level. We disagree, however, that our research framework would not be suitable for the continuous process of rationalization resulting in ever-changing work environments, with a substantial part of these changes being the introduction of ergonomic interventions. When appraising the effects of such ergonomic spin-offs obtained from changes in the production system, a decent scientific funding of the underlying working mechanisms of the intervention (as described in steps 2-3 of our framework) remains imperative. In their conceptualization of the relation between rationalization at societal level and (musculoskeletal) health effects at individual worker level, Westgaard \& Winkel themselves (3) illustrate that the rationalization process at the end of the causal pathway results in changes in exposure to occupational physical and psychosocial risk factors that eventually affect MSD.

Moreover, we also agree that policy measures and interventions at the level of companies or populations may need to be evaluated in ways other than an RCT. In fact, the latter was discussed in the Scandinavian Journal of Work, Environment \& Health (4), where we have listed several alternative observational research designs that may be suitable to evaluate various workplace interventions, such as interrupted time series, regression discontinuity, or multiple baseline design.

Laestadius and colleagues (5) have published an illustrative example of a quasi-experimental research design in the context of MSD prevention. This study evaluated the move of approximately 1500 International Monetary Fund employees to a new office with an improved ergonomics design. In the study, workers who changed offices - which might be called rationalization - were compared to a similar group of World Bank workers that did not move. The study showed a reduction in awkward working postures as well as musculoskeletal pain and eye strain as a result of the change in workplace and individual workstation assessment. We believe that our model is well applicable to a broad range of interventions, such as the one mentioned in this example, as well as adequate methods evaluating the effectiveness of these interventions. In our framework, step 4 (development of intervention(s)), step 5 (evaluation of intervention $(s)$ ) and step 6 (implementation of effective intervention(s)) are suitable to do so and certainly not limited to traditional ergonomic interventions and/or evaluations using RCT only. Despite this, we do believe that, in case an intervention cannot be tested by performing an RCT, we should not neglect the scientific quality limitations of the alternative research designs used. Or as phrased eloquently by Kristensen (6): "There may be many good reasons for not performing a randomized controlled trial in an occupational setting. But there are no good reasons for ignoring the problems created by not applying such a design." As a minimum requirement, we believe that evaluation studies should be longitudinal and should enable the comparison of participants receiving the intervention to an appropriate reference group not receiving the intervention.

In conclusion, we thank Winkel \& Westgaard for their suggestion that a production system's approach, which goes beyond standalone adjustments in the workplace introduced by researchers or ergonomists, can be used to prevent work-related MSD and that we may need alternative research designs to evaluate this approach. We agree with these statements. However, we argue 
that a further development of our research framework, also accommodating interventions introduced by management and other stakeholders in real working life, is unnecessary as our framework already accommodates for this process. Therefore, we invite researchers and ergonomists to fully benefit from the input of corporate stakeholders who aim at organizational sustainability when designing interventions. Researchers should use alternative designs when testing the effectiveness of such interventions if an RCT is not possible and should also prioritize supporting relevant stakeholders in the implementation of effective interventions for the prevention of work-related MSD.

\section{References}

1. Winkel J, Westgaard RH. Development and implementation of interventions managing work-related musculoskeletal disorders: Inadequacy of prevalent research framework and future opportunities. Scand J Work Environ Health. 2019;45(3):316-317. https://doi.org/10.5271/sjweh.3815.

2. van der Beek AJ, Dennerlein JT, Huysmans MA, Mathiassen SE, Burdorf A, van Mechelen W, et al. A research framework for the development and implementation of interventions preventing work-related musculoskeletal disorders. Scand J Work Environ Health 2017 Nov 1;43(6):526-39. https://doi org/10.5271/sjweh.3671.
3. Westgaard RH, Winkel J. Occupational musculoskeletal and mental health: significance of rationalization and opportunities to create sustainable production systems - A systematic review. Appl Ergon 2011 Jan;42(2):261-96. https://doi.org/10.1016/j.apergo.2010.07.002.

4. Schelvis RM, Oude Hengel KM, Burdorf A, Blatter BM, Strijk JE, van der Beek AJ. Evaluation of occupational health interventions using a randomized controlled trial: challenges and alternative research designs. Scand J Work Environ Health 2015 Sep;41(5):491-503. https://doi. org/10.5271/sjweh.3505.

5. Laestadius JG, Ye J, Cai X, Ross S, Dimberg L, Klekner M. The proactive approach--is it worthwhile? A prospective controlled ergonomic intervention study in office workers. J Occup Environ Med 2009 Oct;51(10):1116-24. https://doi. org/10.1097/JOM.0b013e3181bae19d.

6. Kristensen TS. Intervention studies in occupational epidemiology. Occup Environ Med 2005 Mar;62(3):205-10. https://doi.org/10.1136/oem.2004.016097.

\section{Allard J van der Beek, $\mathrm{PhD},{ }^{1}$ and Pieter Coenen, $\mathrm{PhD}^{1}$}

${ }^{1}$ Amsterdam UMC, Vrije Universiteit Amsterdam, Department of Public and Occupational Health, Amsterdam Public Health Research Institute, Amsterdam, The Netherlands.

Correspondence to Prof. Allard van der Beek, Department of Public and Occupational Health, Amsterdam UMC, location VUmc, van der Boechorststraat 7, 1081 BT Amsterdam, The Netherlands. [E-mail: a.vanderbeek@vumc.nl] 